Juarez Ferreira COELHO*

\title{
A inflação baixa distorce, ou não, as demonstrações contábeis
}

\section{Resumo}

Este artigo demonstra, através de um processo de simulação, as distorções causadas pela inflação e os juros embutidos nas transações comerciais das empresas. Hoje, nas demonstrações contábeis elaboradas através das exigências da Lei das S/A's - Lei 6404/76 e o Regulamento do Imposto de Renda - não mais se aplicam as técnicas necessárias para a apuração dos efeitos inflacionários nos demonstrativos contábeis, porque a Lei 9249/95 proibiu a utilização da correção monetária de balanço nos demonstrativos contábeis.

Nos três últimos exercícios, a inflação está relativamente baixa, mas pode provocar grandes distorções na lucratividade e, conseqüentemente, no patrimônio líquido da empresa. Daí a razão da elaboração deste simulado, para averiguação das distorções causadas pelos impactos inflacionários e juros.

\section{Abstract}

This article is a demonstration through a simulation process of distortions caused by inflation and the interests inserted in the commercial transactions. Today, in the accounting demonstrations developed from requirements by Federal Law 6404/ 76 and Income Tax Rules- no more are applied the necessary techniques for verifying the inflationary effects on accounting demonstrations because the Federal Law 9249/95 has prohibited the utilization of monetary amendment for balance in the accounting demonstrations.
In the three last exercises, the inflation is relatively down but it can determine large distortions in the profitableness and, in consequence, in the corporation net assets. To demonstrate this fact by a simulation process was the objective of this article.

Com o advento do Plano Real implantado em nosso país a partir de julho de 1994, sua premissa maior foi tentar eliminar a inflação, ou seja, uma desindexação global da economia. Contudo, podemos observar que esse expurgo só atingiu o lado mais fraco da corda, chamada de pessoa física e jurídica. Conseqüentemente, com a implantação da Lei 9249/95, as empresas foram proibidas de efetuar a correção monetária de balanço; essa nova regra passou a ser praticada já nos Balanços Patrimoniais encerrados a partir de janeiro de 1996.

Se capitalizarmos os índices de inflação medidos pelo IGPM-FGV, iremos detectar um número razoável que, sem dúvida, estará distorcendo as demonstrações contábeis e financeiras, bem como os custos de produção, aumento da carga tributária, através de maior recolhimento de tributos, como: Contribuição Social sobre o Lucro e Imposto de Renda Pessoa Jurídica, distribuição imprópria de dividendos aos sócios e participação nos lucros e resultados dos funcionários.

governo congelou a UFIR (Unidade Fiscal de Referência), índice base que media a inflação, quando não era manipulada pelo mesmo, que acabou sendo extinta como índice para a correção de balanços. Mas, as multas e juros por atraso no pagamento de tributos, para o próprio governo, não foram congelados.

*Doutorando em Controladoria e Contabilidade pela Faculdade de Economia e Administração / USP (aluno especial). Mestre em Ciências Contábeis e Financeiras. Bacharel em Ciências Contábeis. Professor das Faculdades Integradas "Campos Salles" e UNIP. 
A CVM (Comissão de Valores Mobiliários) publicou a Instrução Normativa $64 / 87$, que passou a exigir as demonstrações complementares em moeda de capacidade aquisitiva constante, apenas para as empresas de capital aberto inicialmente, porém, sabemos que a informação contábil elaborada pela sistemática da correção integral, melhora significativamente a demonstração contábil, pois irá refletir a perda ou ganho do poder de compra da moeda, dependendo da estrutura da empresa.

Vimos por vários anos as empresas, que publicam balanços pela sistemática de capacidade aquisitiva constante da moeda, receberem prêmios concedidos pela ANEFAC (Associação Nacional dos Executivos de Finanças, Administração e Contabilidade), pela nobreza da informação contábil.

O contabilista tem o dever de bem informar, para que a direção de sua empresa possa melhor decidir em termos de gestão econômica e financeira.

Este caso prático foi desenvolvido através de dados contábeis hipotéticos, com a utilização da UMC (Unidade Monetária Contábil), criada pela CVM (Comissão de Valores Mobiliários) para ser empregada nas demonstrações elaboradas pela sistemática da correção monetária integral. No exercício desenvolvido, procuramos trabalhar com pequenas variações das UMC's, justamente para detectar distorções entre um resultado elaborado pela legislação societária e pela correção monetária integral.

\begin{tabular}{|l|ll|}
\hline \multicolumn{4}{|c|}{$\begin{array}{c}\text { CASO PRÁTICO DE C.M.I. } \\
\text { EMPRESA INTEGRALIZADORA S/A }\end{array}$} \\
BALANÇO PATRIMONIAL DO EXERCÍCIO ENCERRADO EM $\mathbf{3 1 / 1 2 / 9 6}$ \\
(valores em reais)
\end{tabular}

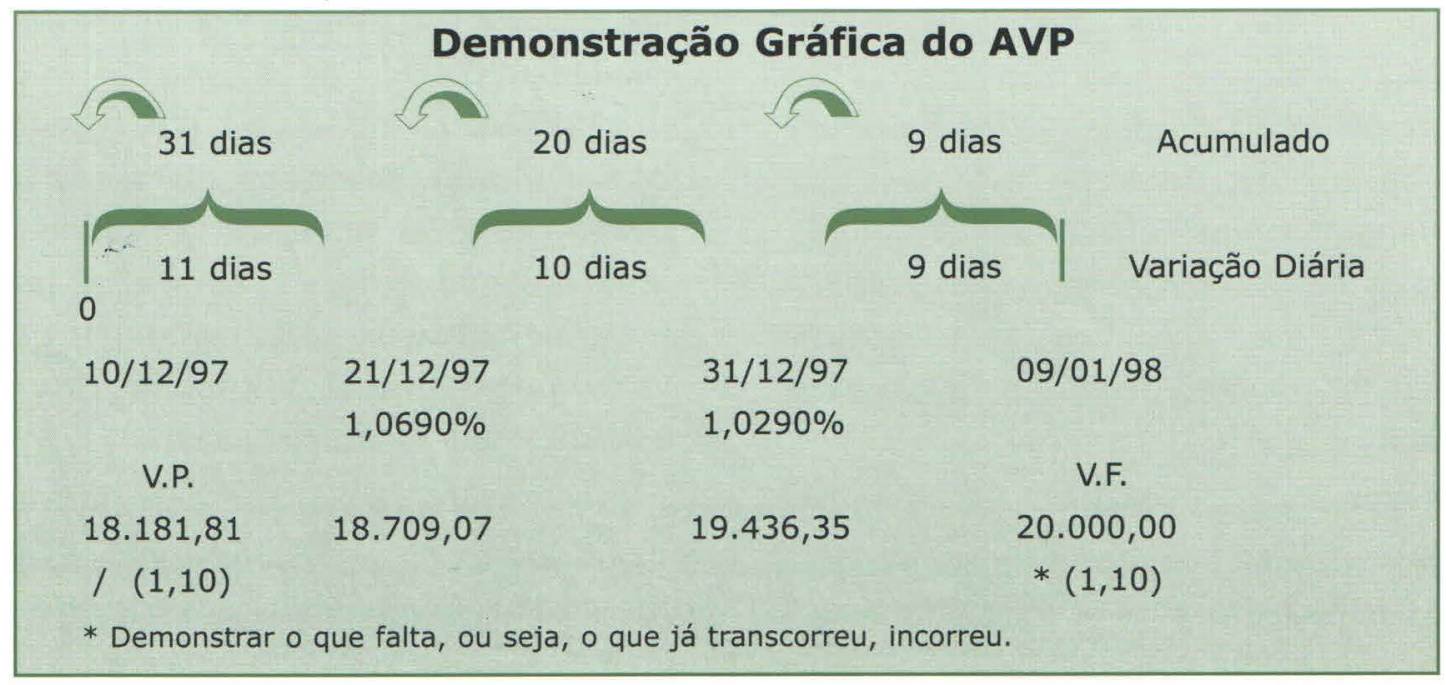




\section{Transações Contábeis} adicionais a serem contabilizadas pela

\section{Societária X Integral}

1. Compra de 20 unidades de mercadorias para revenda, à vista, no valor de $\mathrm{R} \$ 10.000,00$, em 31.01.97;

2. Compra de uma máquina para produção, à vista, no valor de $\mathrm{R} \$ 15.000,00$, em 29.02.97;

3. Apropriação das seguintes despesas em 31.03.97:

- apropriação da Folha de Pagamento no valor de $\mathrm{R} \$ 20.000,00$;

- apropriação dos encargos sociais s/ a folha de pagamento no valor de $\mathrm{R} \$ 7.000,00$;

- apropriação da conta de energia elétrica no valor de $\mathrm{R} \$ 2.500,00$;

- apropriação da conta de água no valor de $\mathrm{R} \$ 2.000,00$;

4. Aquisição de um financiamento de capital de giro no valor de $\mathrm{R} \$ 40.000,00$ em 31.03.97 . ELP;

5. Aquisição de ações de outras empresas, à vista, no valor de $\mathrm{R} \$ 10.000,00$, em 30.04.97;

6. Integralização de capital, mediante investimento direto em moeda no valor de $\mathrm{R} \$$ 20.000,00 em 30.04.97;

7. Depósito judicial efetuado em 31.05.97, referente a inconstitucionalidade do PIS - s/ Faturamento no valor de R\$18.000,00 - RLP;

8. Compra a prazo de estoque de produtos estrangeiros no valor de R\$20.000,00 em 10.12.97, para pagamento em 30 dias; a taxa de juros embutida na transação é de $10 \%$ ao mês, correspondente a 18 unidades;

9. Venda de produtos em 10.12.97, no valor de $\mathrm{R} \$ 50.000,00$ para recebimento em 09.01.98; a taxa de juros embutida na transação é de $12 \%$ ao mês. Baixar do estoque o correspondente a 15 unidades;

10. Apropriação dos seguintes impostos sobre as vendas em 20.12.97:

- PIS s/ faturamento $=0,65 \%-\mathrm{R} \$ 325,00$

- COFINS s/ faturamento $=2,0 \%-\mathrm{R} \$ 1.000,00$

$\cdot$ ICMS s/ faturamento $=18,0 \%-\mathrm{R} \$ 9.000,00$

\section{Informações adicionais}

A- Elaborar todos os razonetes das transações acima, e dos saldos iniciais, pela legislação societária e para fins da integral em UMC's.

B- Calcular todos os ganhos e perdas monetárias ocorridos nas transações.

C- Elaborar todas as demonstrações contábeis pela legislação societária X integral.

D- As demonstrações finais do exercício serão elaboradas no dia 31.12.97.

BP, DRE, DMPL - Conciliação do Lucro Líquido, RAZUMC, Ficha de Estoque.

E- Os valores das UMC's a serem utilizadas no exercício são as seguintes:

\begin{tabular}{|cc|}
\hline PERÍODO & VLR. UMC \\
31.12 .96 & 0,8287 \\
31.01 .97 & 0,8453 \\
29.02 .97 & 0,8877 \\
31.03 .97 & 0,9143 \\
30.04 .97 & 0,9509 \\
31.05 .97 & 0,9889 \\
31.10 .97 & 1,0285 \\
30.11 .97 & 1,0594 \\
10.12 .97 & 1,1018 \\
20.12 .97 & 1,1215 \\
31.12 .97 & 1,1500 \\
\hline
\end{tabular}

F- Contabilizar a depreciação sobre o imobilizado à taxa de $10 \%$ ao ano, considerar proporcionalmente.

G- Efetuar a correção monetária de balanço, para fins da correção monetária integral.

H- Calcular a provisão para Contribuição Social s/ o Lucro e IRPJ à taxa de $10 \%$ e $15 \%$ respectivamente. 
Passos para o desenvolvimento do exercício

1. Contabilizar todas as transações pela legislação societária em razonetes.

2. Elaborar a ficha de estoque pela legislação societária $\mathrm{x}$ integral.

3. Elaborar todas as demonstrações contábeis e financeiras pela legislação societária.

4. Elaborar o RAZUMC (Razão Auxiliar em UMC's) para fins de ajuste da correção monetária de balanço na Integral.

5. Contabilizar todas as transações em UMC's, para fins da integral.

6. Efetuar o cálculo dos ajustes a valor presente, das transações mercantis a prazo.

7. Elaborar as demonstrações contábeis pela Integral.

8. Elaborar a conciliação do prejuízo líquido do exercício pela legislação societária e integral, para justificar a diferença entre os prejuízos obtidos.

Empresa integralizadora S/A

Verificação em razonetes pela

Legislação Societária

LEI 6.404/76

\begin{tabular}{|ll|rr|}
\hline \multicolumn{4}{|c|}{ BANCOS CONTA MOVIMENTO } \\
\hline SI & $80.000,00$ & $10.000,00$ & $\mathbf{1}$ \\
$\mathbf{4}$ & $40.000,00$ & $15.000,00$ & $\mathbf{2}$ \\
$\mathbf{6}$ & $20.000,00$ & $10.000,00$ & $\mathbf{5}$ \\
& & $18.000,00$ & $\mathbf{7}$ \\
\hline & $140.000,00$ & $53.000,00$ & \\
\hline SF & $\mathbf{8 7 . 0 0 0 , 0 0}$ & \\
\hline
\end{tabular}

CAPITAL INTEGRALIZADO

$80.000,00$ SI

$20.000,00 \quad 6$

$100.000,00 \mathrm{SF}$

\begin{tabular}{|ll|cl|}
\hline \multicolumn{4}{|c|}{ ESTOQUES } \\
\hline $\mathbf{1}$ & $10.000,00$ & $11.842,10$ & $\mathbf{9}$ \\
$\mathbf{8}$ & $20.000,00$ & & \\
\hline & $30.000,00$ & $11.842,10$ \\
\hline SF & $\mathbf{1 8 . 1 5 7 , 9 0}$ & & \\
\hline
\end{tabular}

\begin{tabular}{|ll|l|}
\hline \multicolumn{3}{|c|}{ MÁQ E EQUIPAMENTOS } \\
\hline $\mathbf{2}$ & $15.000,00$ & \\
\hline SF & $\mathbf{1 5 . 0 0 0 , 0 0}$ & \\
\hline
\end{tabular}

SALÁRIOS A PAGAR

$20.000,00$ 3A

$\mathbf{2 0 . 0 0 0 , 0 0 ~ S F ~}$

\begin{tabular}{|ll|r|}
\hline \multicolumn{3}{|c|}{ DESPESAS COM SALÁRIOS } \\
\hline 3A & $20.000,00$ & \\
\hline SF & $\mathbf{2 0 . 0 0 0 , 0 0}$ & $20.000,00$ ARE1 \\
\hline
\end{tabular}

\begin{tabular}{|c|c|c|}
\hline \multicolumn{3}{|c|}{ DEPRECIAÇÃO ACUMULADA } \\
\hline & $1.250,00$ & $\mathbf{F}$ \\
\hline & $1.250,00$ & SF \\
\hline
\end{tabular}

\begin{tabular}{|lc|r|}
\hline \multicolumn{3}{|c|}{ DESPESAS COM DEPRECIAÇÃO } \\
\hline F & $1.250,00$ & \\
\hline SF & $\mathbf{1 . 2 5 0 , 0 0}$ & $1.250,00$ ARE2 \\
\hline
\end{tabular}

ENCARGOS SOCIAIS A RECOLHER

$7.000,00$ 3B

$7.000,00$ SF

DESPESAS COM ENCARGOS SOCIAIS

\begin{tabular}{|ll|l|}
\hline 3B & $7.000,00$ & \\
\hline SF & $\mathbf{7 . 0 0 0 , 0 0}$ & $7.000,00$ ARE3 \\
\hline
\end{tabular}

ENERGIA ELÉTRICA A PAGAR

$2.500,00 \quad 3 \mathrm{C}$

$2.500,00 \mathrm{SF}$ 


\begin{tabular}{|c|c|c|}
\hline \multicolumn{3}{|c|}{ DESPESAS COM ENERGIA ELÉTRICA } \\
\hline $3 C$ & $2.500,00$ & \\
\hline SF & $2.500,00$ & 2.500,00 ARE4 \\
\hline
\end{tabular}

\begin{tabular}{|l|cc|}
\hline \multicolumn{3}{|c|}{ CONTA DE ÁGUA A PAGAR } \\
\hline & $2.000,00$ & 3D \\
\hline & $2.000,00$ & SF \\
\hline
\end{tabular}

\begin{tabular}{|ll|r|}
\hline \multicolumn{3}{|c|}{ DESPESAS COM ÁGUA } \\
\hline 3D & $2.000,00$ & \\
\hline SF & $\mathbf{2 . 0 0 0 , 0 0}$ & $2.000,00$ ARE5 \\
\hline
\end{tabular}

FINANC. CAP. GIRO - ELP

$\begin{array}{rr}40.000,00 & 4 \\ \mathbf{4 0 . 0 0 0 , 0 0} & \text { SF }\end{array}$

\begin{tabular}{|l|l|l|}
\hline \multicolumn{3}{|c|}{ AÇÕES DE OUTRAS EMPRESAS - INV } \\
\hline $\mathbf{5}$ & $10.000,00$ & \\
\hline SF & $\mathbf{1 0 . 0 0 0 , 0 0}$ & \\
\hline
\end{tabular}

\begin{tabular}{|ll|l|}
\hline \multicolumn{3}{|c|}{ DEPÓSITOS JUDICIAIS - RLP } \\
\hline $\mathbf{7}$ & $18.000,00$ & \\
\hline SF & $\mathbf{1 8 . 0 0 0 , 0 0}$ & \\
\hline
\end{tabular}

\begin{tabular}{|c|c|c|}
\hline \multicolumn{3}{|c|}{ PIS SOBRE FATURAMENTO } \\
\hline 10A & 325,00 & \\
\hline SF & 325,00 & 325,00 ARE6 \\
\hline
\end{tabular}

\begin{tabular}{|l|rl|}
\hline \multicolumn{3}{|c|}{ FORNECEDOR ESTRANGEIRO } \\
\hline & $20.000,00$ & $\mathbf{8}$ \\
\hline & $\mathbf{2 0 . 0 0 0 , 0 0}$ & SF \\
\hline
\end{tabular}

\begin{tabular}{|lc|r|}
\hline \multicolumn{3}{|c|}{ CUSTO DOS PRODUTOS VENDIDOS } \\
\hline $\mathbf{9}$ & $11.842,10$ & \\
\hline SF & $\mathbf{1 1 . 8 4 2 , 1 0}$ & $11.842,10$ ARE7 \\
\hline
\end{tabular}

\begin{tabular}{|c|c|c|}
\hline \multicolumn{3}{|c|}{ COFINS SOBRE FATURAMENTO } \\
\hline 10B & $1.000,00$ & \\
\hline & $1.000,00$ & 1.000,00 ARE8 \\
\hline
\end{tabular}

\begin{tabular}{|c|c|c|}
\hline \multicolumn{3}{|c|}{ ICMS SOBRE FATURAMENTO } \\
\hline 10C & $9.000,00$ & \\
\hline SF & $9.000,00$ & $9.000,00$ ARE9 \\
\hline
\end{tabular}

\begin{tabular}{|ll|ll|}
\hline \multicolumn{4}{|c|}{ RECEITA BRUTA DE VENDAS } \\
\hline & & $50.000,00$ & 9 \\
\hline ARE10 & $50.000,00$ & $50.000,00$ & SF \\
\hline
\end{tabular}

\begin{tabular}{|cl|l|}
\hline \multicolumn{3}{|c|}{ CLIENTES } \\
\hline $\mathbf{9}$ & $50.000,00$ & \\
\hline SF & $\mathbf{5 0 . 0 0 0 , 0 0}$ & \\
\hline
\end{tabular}

\begin{tabular}{|r|rr|}
\hline \multicolumn{3}{|c|}{ IMPOSTO A PAGAR } \\
\hline & 325,00 & $\mathbf{1 0 A}$ \\
& $1.000,00$ & $\mathbf{1 0 B}$ \\
& $9.000,00$ & $\mathbf{1 0 C}$ \\
\hline & $\mathbf{1 0 . 3 2 5 , 0 0}$ & SF \\
\hline
\end{tabular}

\begin{tabular}{|l|l|}
\hline \multicolumn{3}{|c|}{ PREJUÍzO ACUMULADO } \\
\hline TRF1 $4.917,10$ & \\
\hline
\end{tabular}

\begin{tabular}{|lr|r|}
\hline \multicolumn{3}{|c|}{ APURAÇÃO DE RESULTADO DO EXERC. } \\
\hline ARE1 & $20.000,00$ & $50.000,00$ ARE \\
ARE2 & $1.250,00$ & \\
ARE3 & $7.000,00$ & \\
ARE4 & $2.500,00$ & \\
ARE5 & $2.000,00$ & \\
ARE6 & 325,00 & \\
ARE7 & $11.842,10$ & \\
ARE8 & $1.000,00$ & \\
ARE9 & $9.000,00$ & \\
\hline \multicolumn{3}{|c|}{} \\
\end{tabular}


Verificação em razonetes pela Correção Monetária Integral Em UMC'S

\begin{tabular}{|lr|lr|}
\hline \multicolumn{4}{|c|}{ BANCOS CONTA MOVIMENTO } \\
\hline SI & $96.536,7443$ & $11.830,1195$ & $\mathbf{1}$ \\
$\mathbf{4}$ & $43.749,3164$ & $16.897,6005$ & $\mathbf{2}$ \\
$\mathbf{6}$ & $21.032,7059$ & $10.516,3529$ & $\mathbf{5}$ \\
& & $18.202,0427$ & $\mathbf{7}$ \\
\hline & $161.318,7666$ & $57.446,1156$ & \\
\hline & $\mathbf{1 0 3 . 8 7 2 , 6 5 1 0}$ & $28.221,4601$ & PM1 \\
\hline SF & $\mathbf{7 5 . 6 5 1 , 1 9 0 9}$ & & \\
& $X 1,1500$ & & \\
& R $\$ 87.000,00$ & & \\
\hline
\end{tabular}

\begin{tabular}{|r|rr|}
\hline \multicolumn{3}{|c|}{ CAPITAL INTEGRALIZADO } \\
\hline & $96.536,7443$ & SI \\
& $21.032,7059$ & $\mathbf{6}$ \\
\hline & $\mathbf{1 1 7 . 5 6 9 , 4 5 0 2}$ & SF \\
$\times 1,1500$ & \\
& $\mathrm{R} \$ 135.204,87$ & \\
\hline
\end{tabular}

\begin{tabular}{|lr|r|}
\hline \multicolumn{4}{|c|}{ ESTOQUES } \\
\hline $\mathbf{1}$ & $11.830,1195$ & $11.183,7000$ \\
$\mathbf{8}$ & $16.501,9150$ & $\mathbf{9}$ \\
\hline & $28.332,0345$ & $11.183,7000$ \\
\hline SF & $\mathbf{1 7 . 1 4 8 , 3 3 4 5}$ & \\
& $\times 1,1500$ & \\
& $\mathrm{R} \$ 19.720,58$ & \\
\hline
\end{tabular}

\begin{tabular}{|c|c|c|}
\hline \multicolumn{3}{|c|}{ MÁQ E EQUIPAMENTOS } \\
\hline 2 & $16.897,6005$ & \\
\hline SF & $16.897,6005$ & \\
\hline & $\times 1,1500$ & \\
\hline & $\mathrm{R} \$ 19.432,24$ & \\
\hline
\end{tabular}

\begin{tabular}{|rr|rr|}
\hline \multicolumn{4}{|c|}{ SALÁRIOS A PAGAR } \\
\hline & & $21.874,6582$ & 3A \\
\hline GM1 & $4.483,3500$ & $\mathbf{2 1 . 8 7 4 , 6 5 8 2}$ & \\
\hline & & $17.391,3082$ & SF \\
& & $\times 1,1500$ & \\
& & $R \$ 20.000,00$ & \\
\hline
\end{tabular}

\begin{tabular}{|rr|rr|}
\hline \multicolumn{4}{|c|}{ DESPESAS COM SALÁRIOS } \\
\hline 3A & $21.874,6582$ & & \\
\hline & $\mathbf{2 1 . 8 7 4 , 6 5 8 2}$ & $4.483,3500$ & GM1 \\
\hline SF & $\mathbf{1 7 . 3 9 1 , 3 0 8 2}$ & $\mathbf{1 7 . 3 9 1 , 3 0 8 2}$ & ARE1 \\
$\times 1,1500$ & & \\
& R $\$ 20.000,00$ & & \\
\hline \multicolumn{4}{|c|}{} \\
\hline \multicolumn{4}{|c|}{ DEPRECIAÇÃO ACUMULADA } \\
\hline & $1.408,1334$ & F \\
& $\mathbf{1 . 4 0 8 , 1 3 3 4}$ & SF \\
\hline & $X 1,1500$ & \\
\hline
\end{tabular}

\begin{tabular}{|lr|r|}
\hline \multicolumn{3}{|c|}{ DESPESAS COM DEPRECIAÇÃO } \\
\hline F & $1.408,1334$ & \\
\hline SF & $\mathbf{1 . 4 0 8 , 1 3 3 4}$ & $1.408,1334$ ARE2 \\
\hline & $\times 1,1500$ & \\
& R $\$ 1.619,35$ & \\
\hline
\end{tabular}

ENCARGOS SOCIAIS A RECOLHER

\begin{tabular}{|rr|rr|}
\hline & $7.656,1304$ & 3B \\
\hline GM2 & $1.569,1700$ & $\mathbf{7 . 6 5 6 , 1 3 0 4}$ & \\
\hline & & $\mathbf{6 . 0 8 6 , 9 6 0 4}$ & SF \\
& $\times 1,1500$ & \\
& $R \$ 7.000,00$ & \\
\hline
\end{tabular}

DESPESAS COM ENCARGOS SOCIAIS

\begin{tabular}{|rr|rr|}
\hline 3B & $7.656,1304$ & & \\
\hline & $\mathbf{7 . 6 5 6 , 1 3 0 4}$ & $1.569,1700$ & GM2 \\
\hline SF & $\mathbf{6 . 0 8 6 , 9 6 0 4}$ & $\mathbf{6 . 0 8 6 , 9 6 0 4}$ & ARE3 \\
& $\times 1,1500$ & & \\
& $R \$ 7.000,00$ & & \\
\hline
\end{tabular}

\begin{tabular}{|cc|cc|}
\hline \multicolumn{3}{|c|}{ ENERGIA ELÉTRICA A PAGAR } \\
\hline & & $2.734,3323$ & 3C \\
\hline GM3 & 560,4100 & $\mathbf{2 . 7 3 4 , 3 3 2 3}$ & \\
\hline & & $\mathbf{2 . 1 7 3 , 9 2 2 3}$ & SF \\
& & $\times 1,1500$ & \\
& & $R \$ 2.500,00$ & \\
\hline
\end{tabular}




\begin{tabular}{|r|r|r|r|}
\hline \multicolumn{4}{|c|}{ DESPESAS COM ENERGIA ELÉTRICA } \\
\hline 3C & $2.734,3323$ & & \\
\hline & $\mathbf{2 . 7 3 4 , 3 3 2 3}$ & 560,4100 & GM3 \\
\hline SF & $\mathbf{2 . 1 7 3 , 9 2 2 3}$ & $\mathbf{2 . 1 7 3 , 9 2 2 3}$ & ARE4 \\
& $\times 1,1500$ & & \\
R $\$ 2.500,00$ & & \\
\hline
\end{tabular}

\begin{tabular}{|c|c|c|c|}
\hline \multicolumn{4}{|c|}{ CONTA DE ÁGUA A PAGAR } \\
\hline & & $2.187,4658$ & 3D \\
\hline GM4 & 448,3300 & $2.187,4658$ & \\
\hline & & $\begin{array}{r}1.739,1358 \\
\times 1,1500 \\
R \$ 2.000,00\end{array}$ & SF \\
\hline
\end{tabular}

\begin{tabular}{|rr|rr|}
\hline \multicolumn{4}{|c|}{ DESPESAS COM ÁGUA } \\
\hline 3D & $2.187,4658$ & & \\
\hline & $\mathbf{2 . 1 8 7 , 4 6 5 8}$ & 448,3300 & GM4 \\
\hline SF & $\mathbf{1 . 7 3 9 , 1 3 5 8}$ & $\mathbf{1 . 7 3 9 , 1 3 5 8}$ & ARE5 \\
& $\times 1,1500$ & & \\
& R $\$ 2.000,00$ & & \\
\hline
\end{tabular}

\begin{tabular}{|rr|rr|}
\hline \multicolumn{3}{|c|}{ FINANC. CAP. GIRO - ELP } \\
\hline & & $43.749,3164$ & $\mathbf{4}$ \\
\hline GM5 & $8.966,7000$ & $\mathbf{4 3 . 7 4 9 , 3 1 6 4}$ & \\
\hline & & $\mathbf{3 4 . 7 8 2 , 6 1 6 4}$ & SF \\
& & $\times 1,1500$ & \\
& & R $\$ 40.000,00$ & \\
\hline
\end{tabular}

\begin{tabular}{|lr|r|}
\hline \multicolumn{3}{|c|}{ AÇÕES OUTRAS EMPRESAS - INV } \\
\hline $\mathbf{5}$ & $10.516,3529$ & \\
\hline SF & $\mathbf{1 0 . 5 1 6 , 3 5 2 9}$ & \\
\hline & $\times 1,1500$ & \\
& $\mathrm{R} \$ 12.093,81$ & \\
\hline
\end{tabular}

\begin{tabular}{|lr|c|}
\hline \multicolumn{3}{|c|}{ DEPÓSITOS JUDICIAIS - RLP } \\
\hline $\mathbf{7}$ & $18.202,0427$ & $2.549,8600$ \\
\hline SF & $\mathbf{1 5 . 6 5 2 , 1 8 2 7}$ & \\
\hline & PM2 \\
\hline & R 1,1500 & \\
& $18.000,00$ & \\
\hline
\end{tabular}

\begin{tabular}{|l|r|r|r|}
\hline \multicolumn{4}{|c|}{ PIS SOBRE FATURAMENTO } \\
\hline 10A & 289,7905 & 7,1800 & GM6 \\
\hline SF & $\mathbf{2 8 2 , 6 1 0 5}$ & $\mathbf{2 8 2 , 6 1 0 5}$ & ARE6 \\
\hline & $X 1,1500$ & & \\
& R $\$ 325,00$ & & \\
\hline
\end{tabular}

\begin{tabular}{|c|c|c|c|}
\hline \multicolumn{4}{|c|}{ FORNECEDOR ESTRANGEIRO } \\
\hline & & $16.501,9150$ & 8 \\
\hline & & $16.501,9150$ & SF \\
\hline \multirow[t]{4}{*}{ GM7 } & 737,0800 & $1.038,5000$ & AVP1 \\
\hline & & $16.803,3350$ & \\
\hline & & $\times 1,1500$ & \\
\hline & & $\mathrm{R} \$ 19.323,84$ & \\
\hline
\end{tabular}

\begin{tabular}{|lr|r|r|}
\hline \multicolumn{4}{|c|}{ CUSTO DOS PROD. VENDIDOS } \\
\hline $\mathbf{9}$ & $11.183,7000$ & & \\
\hline SF & $\mathbf{1 1 . 1 8 3 , 7 0 0 0}$ & $\mathbf{1 1 . 1 8 3 , 7 0 0 0}$ & ARE7 \\
\hline & X 1,1500 & & \\
& R $\$ 12.861,26$ & & \\
\hline
\end{tabular}

\begin{tabular}{|lr|rr|}
\hline \multicolumn{4}{|c|}{ COFINS SOBRE FATURAMENTO } \\
\hline 10B & 891,6630 & 22,1000 & GM8 \\
\hline SF & $\mathbf{8 6 9 , 5 6 3 0}$ & $\mathbf{8 6 9 , 5 6 3 0}$ & ARE8 \\
\hline & & $\times 1,1500$ & \\
& & $R \$ 1.000,00$ & \\
\hline
\end{tabular}

\begin{tabular}{|lr|rr|}
\hline \multicolumn{4}{|c|}{ ICMS SOBRE FATURAMENTO } \\
\hline 10C & $8.024,9666$ & 198,8800 & GM9 \\
\hline SF & $\mathbf{7 . 8 2 6 , 0 8 6 6}$ & $\mathbf{7 . 8 2 6 , 0 8 6 6}$ & ARE9 \\
\hline & $X 1,1500$ & & \\
& R $\$ 9.000,00$ & & \\
\hline
\end{tabular}

\begin{tabular}{|l|rr|}
\hline \multicolumn{3}{|c|}{ RECEITA BRUTA DE VENDAS } \\
\hline & $40.518,1067$ & $\mathbf{9}$ \\
\hline ARE10 40.518,1067 & $\mathbf{4 0 . 5 1 8 , 1 0 6 7}$ & SF \\
\hline & $X 1,1500$ & \\
& $R \$ 46.595,82$ & \\
\hline
\end{tabular}




\begin{tabular}{|lr|rr|}
\hline \multicolumn{4}{|c|}{ CLIENTES } \\
\hline $\mathbf{9}$ & $40.518,1067$ & $1.838,4523$ & PM2 \\
\hline AVP2 & $3.205,1652$ & $1.838,4523$ & \\
\hline SF & $\mathbf{4 1 . 8 8 4 , 8 1 9 6}$ & & \\
& $\times 1,1500$ & & \\
& $\mathrm{R} \$ 48.167,54$ & & \\
\hline
\end{tabular}

\begin{tabular}{|lr|r|}
\hline \multicolumn{3}{|c|}{ DESPESAS FINANCEIRAS } \\
\hline PM1 & $28.220,4600$ & $8.966,7000$ \\
PM2 & $2.549,8600$ & \\
\hline & $30.770,3200$ & $8.966,7000$ \\
\hline SF & $\mathbf{2 1 . 8 0 3 , 6 2 0 0}$ & $\mathbf{2 1 . 8 0 3 , 6 2 0 0}$ \\
& $\times 1,1500$ & \\
& R $\$ 25.074,16$ & \\
\hline
\end{tabular}

\begin{tabular}{|lr|rr|}
\hline \multicolumn{4}{|c|}{ IMPOSTOS A PAGAR } \\
\hline GM6 & 7,1800 & 289,7905 & 10A \\
GM8 & 22,1000 & 891,6630 & 10B \\
GM9 & 198,8800 & $8.024,9666$ & 10C \\
\hline & $\mathbf{2 2 8 , 1 6 0 0}$ & $\mathbf{9 . 2 0 6 , 4 2 0 1}$ & \\
\hline & & $\mathbf{8 . 9 7 8 , 2 6 0 1}$ & SF \\
& & $X 1,1500$ & \\
& & $R \$ 10.325,00$ & \\
\hline
\end{tabular}

\begin{tabular}{|lr|r|r|}
\hline \multicolumn{3}{|c|}{ DESPESAS FINANCEIRAS COM. LÍQUIDA } \\
\hline AVP1 & $1.038,5000$ & 737,0800 & GM7 \\
\hline SF & $\mathbf{3 0 1 , 4 2 0 0}$ & $\mathbf{3 0 1 , 4 2 0 0}$ & ARE12 \\
& $X 1,1500$ & \\
& R $\$ 346,63$ & \\
\hline
\end{tabular}

\begin{tabular}{|r|r|}
\hline \multicolumn{2}{|c|}{ PREJUÍZO ACUMULADO } \\
\hline TRF1 $21.886,2281$ \\
$\times 1,1500$ \\
R\$ $25.169,15$ \\
\hline
\end{tabular}

\begin{tabular}{|c|c|c|c|}
\hline \multicolumn{4}{|c|}{ APURAÇÃO DE RESULTADO DO EXERC. } \\
\hline ARE1 & $17.391,3082$ & $40.518,1100$ & ARE10 \\
\hline ARE2 & $1.408,1334$ & & \\
\hline ARE3 & $6.086,9604$ & & \\
\hline ARE4 & $2.173,9223$ & & \\
\hline ARE5 & $1.739,1358$ & & \\
\hline ARE6 & 282,6105 & & \\
\hline ARE7 & $11.183,7000$ & & \\
\hline ARE8 & 869,5630 & & \\
\hline ARE9 & $7.826,0866$ & & \\
\hline ARE11 & $21.803,6200$ & & \\
\hline ARE12 & 301,4200 & $1.366,7129$ & ARE13 \\
\hline & $71.066,4602$ & $41.884,8229$ & \\
\hline \multirow{6}{*}{\multicolumn{2}{|c|}{$29.181,6373$}} & & \\
\hline & & $2.918,1637$ & CSLL \\
\hline & & $4.377,2455$ & IRPJ \\
\hline & & $21.886,2281$ & TRF1 \\
\hline & & $\times 1,1500$ & \\
\hline & & $R \$ 25.169,15$ & \\
\hline
\end{tabular}

RECEITA FINANCEIRA COM. LÍQUIDA

\begin{tabular}{|ll|rr|}
\hline PM2 & $1.838,4523$ & $3.205,1652$ & AVP2 \\
\hline ARE13 & $\mathbf{1 . 3 6 6 , 7 1 2 9}$ & $\mathbf{1 . 3 6 6 , 7 1 2 9}$ & SF \\
& & $\times 1,1500$ & \\
& & $R \$ 1.5716,72$ & \\
\hline
\end{tabular}

\section{CONTRIBUIÇÃO SOCIAL DIFERIDA}

CSSL $2.918,1637$

SF $2.918,1637$

$\times 1,1500$

$\mathrm{R} \$ 3.355,88$
IMPOSTO DE RENDA DIFERIDO

\begin{tabular}{|lr|}
\hline IRPJ & $4.377,2455$ \\
\hline SF & $4.377,2455$ \\
& $\times 1,1500$ \\
& $R \$ 5.033,83$
\end{tabular}


Empresa integralizadora S/A

Balanço patrimonial do exercício encerrado em 31.12.97

(Valores em Reais)

\begin{tabular}{|c|c|c|c|c|c|}
\hline ATIVO & $\begin{array}{r}\text { LEG. SOC. } \\
31.12 .97\end{array}$ & $\begin{array}{r}\text { C.M.I } \\
31.12 .97\end{array}$ & PASSIVO & $\begin{array}{r}\text { LEG. SOC. } \\
31.12 .97\end{array}$ & $\begin{array}{r}\text { C.M.I. } \\
31.12 .97\end{array}$ \\
\hline Circulante & & & Circulante & & \\
\hline Banco cta Movimento & $87.000,00$ & $87.000,00$ & Contas a Pagar & $4.500,00$ & $4.500,00$ \\
\hline Cliente & $50.000,00$ & $48.167,54$ & Salários + Encargos a Pag & $27.000,00$ & $27.000,00$ \\
\hline Estoque & $18.157,90$ & $19.720,58$ & Impostos a Pagar & $10.325,00$ & $10.325,00$ \\
\hline Contribuição Social Diferida & 0,00 & $3.355,88$ & Fornecedor Estrangeiro & $20.000,00$ & $19.323,81$ \\
\hline \multirow[t]{2}{*}{ Imposto de Renda Diferido } & 0,00 & $5.033,83$ & & $61.825,00$ & $61.148,81$ \\
\hline & $155.157,90$ & $163.277,83$ & & & \\
\hline \multicolumn{3}{|l|}{ Realizável a Longo Prazo } & \multicolumn{3}{|l|}{ Exigível a Longo Prazo } \\
\hline \multirow[t]{2}{*}{ Depósito Judicial - PIS } & $18.000,00$ & $18.000,00$ & \multirow[t]{2}{*}{ Financiamento Cap. Giro } & $40.000,00$ & $40.000,00$ \\
\hline & $18.000,00$ & $18.000,00$ & & $40.000,00$ & $40.000,00$ \\
\hline Permanente & & & Patrimônio Líquido & & \\
\hline Investimento & $10.000,00$ & $12.093,81$ & Capital Integ./Atualizado & $100.000,00$ & $135.204,87$ \\
\hline Imobilizado - Custo Corrigido & to $15.000,00$ & $19.432,24$ & Reserva de Lucro & 0,00 & 0,00 \\
\hline \multirow[t]{2}{*}{ (-) Depreciação Acumulada } & $(1.250,00)$ & $(1.619,35)$ & Prejuízo Acumulado & $(4.917,10)$ & $(25.169,15)$ \\
\hline & $23.750,00$ & $29.906,70$ & & $95.082,90$ & $110.035,72$ \\
\hline TOTAL DO ATIVO & $196.907,90$ & $211.184,53$ & TOTAL DO PASSIVO & $196.907,90$ & $211.184,53$ \\
\hline
\end{tabular}

Empresa integralizadora S/A

Demonstração de resultado do exercício encerrado em 31.12.97

(Valores em Reais)

\begin{tabular}{|lrr|}
\hline & LEG.SOC & \multicolumn{1}{c|}{ C.M.I. } \\
Receita Bruta de Vendas & $\mathbf{R} \mathbf{\$}$ & $\mathbf{R 4}$ \\
(-) Impostos Incidentes sobre Vendas & $\mathbf{5 0 . 0 0 0 , 0 0}$ & $\mathbf{4 6 . 5 9 5 , 8 2}$ \\
(=) Receita Líquida de Vendas & $(10.325,00)$ & $(10.325,00)$ \\
(-) Custo dos Produtos Vendidos & $\mathbf{3 9 . 6 7 5 , 0 0}$ & $\mathbf{3 6 . 2 7 0 , 8 2}$ \\
(=) Lucro Bruto & $(11.842,10)$ & $(12.861,26)$ \\
(-) Despesas Administrativas (salários + encargos soc) & $\mathbf{2 7 . 8 3 2 , 9 0}$ & $\mathbf{2 3 . 4 0 9 , 5 6}$ \\
(-) Despesas Financeiras & $(27.000,00)$ & $(27.000,00)$ \\
(-) Depreciações & 0,00 & $(25.074,16)$ \\
(-) Outras Desp. Operac. (Água, Energ., Outras Ctas.) & $(1.250,00)$ & $(1.619,35)$ \\
(=) Lucro Operacional & $(4.500,00)$ & $(4.500,00)$ \\
(+) Receita Financeira Comercial Líquida & $\mathbf{( 4 . 9 1 7 , 1 0 )}$ & $\mathbf{( 3 4 . 7 8 3 , 9 5 )}$ \\
(-) Despesa Financeira Comercial Líquida & 0,00 & $1.571,72$ \\
(-) Resultado Devedor da Corr. Monetária de Balanço & 0,00 & $(346,63)$ \\
(=) Prejuízo antes da CSLL e IRPJ & 0,00 & 0,00 \\
(+) Contribuição Social S/ o Lucro Diferida & $\mathbf{( 4 . 9 1 7 , 1 0 )}$ & $\mathbf{( 3 3 . 5 5 8 , 8 6 )}$ \\
(+) Imposto de Renda Diferido & 0,00 & $3.355,88$ \\
(=) Prejuízo Líquido do Exercício & 0,00 & $5.033,83$ \\
\hline
\end{tabular}


Empresa integralizadora S/A

Demonstração das mutações do patrimônio líquido

do exercício encerrado em 31.12.97 - Societária

(Valores em Reais)

\begin{tabular}{|l|r|r|r|r|r|}
\hline \multicolumn{1}{|c|}{ Discriminação } & $\begin{array}{c}\text { Capital } \\
\text { Integral }\end{array}$ & $\begin{array}{c}\text { Reserva da } \\
\text { C.M. Capital }\end{array}$ & $\begin{array}{c}\text { Reserva } \\
\text { de Lucros }\end{array}$ & $\begin{array}{c}\text { Prejuízos } \\
\text { Acumulados }\end{array}$ & Total \\
\hline \begin{tabular}{l|r|r|r|} 
Saldo em 31/12/96 \\
Aumento de Capital em R\$
\end{tabular} & $\begin{array}{r}80.000,00 \\
\text { Correção Monetária }\end{array}$ & 0 & 0 & 0,00 & $80.000,00$ \\
\hline $\begin{array}{l}\text { Prejuízo Líquido do Exercício } \\
\text { Saldo em 31 / 12 / } 97\end{array}$ & 0,00 & 0 & 0 & 0,00 & $20.000,00$ \\
\hline
\end{tabular}

Empresa integralizadora S/A

Demonstração das mutações do patrimônio líquido do exercício encerrado em 31.12.97 - C.M.I.

(Valores em Reais)

\begin{tabular}{|c|c|c|c|c|c|}
\hline Discriminação & $\begin{array}{c}\text { Capital } \\
\text { Atualizado }\end{array}$ & $\begin{array}{l}\text { Reserva da } \\
\text { C.M. Capital }\end{array}$ & $\begin{array}{c}\text { Reserva } \\
\text { de Lucros }\end{array}$ & $\begin{array}{c}\text { Prejuízo } \\
\text { Acumulado }\end{array}$ & Total \\
\hline Saldo em $31 / 12 / 96$ & $80.000,00$ & 0 & 0 & 0,00 & $80.000,00$ \\
\hline Aumento de Capital $R \$$ & $20.000,00$ & & & & $20.000,00$ \\
\hline Correção Monetária & $35.204,87$ & 0 & 0 & 0,00 & $35.204,87$ \\
\hline Prejuízo Líquido do Exercício & & & & $(25.169,15)$ & $(25.169,15)$ \\
\hline Saldo em 31/12/97 & $135.204,87$ & 0 & 0 & $(25.169,15)$ & $110.035,72$ \\
\hline
\end{tabular}

Empresa integralizadora S/A

Conciliação do lucro líquido do exercício

(Legislação Societária X Correção Monetária Integral)

(Valores em Reais)

\begin{tabular}{|lr|}
\hline & \multicolumn{1}{|c|}{$\mathbf{3 1 . 9 7}$} \\
Prejuízo Líquido do Exercício Pela Legislação Societária & $\mathbf{R . 9 1 7 , 1 0}$ \\
(+) Efeito da Correção de Balanço não reconhecida no Societário & $(29.048,17)$ \\
(+) Atualização Monetária dos estoques & $1.562,68$ \\
(+) AVP - Fornecedor & 676,19 \\
(-) AVP - Clientes & $(1.832,46)$ \\
(+) Contribuição Social s/ o Lucro Diferida & $3.355,88$ \\
(+) Imposto de Renda Diferido & $5.033,83$ \\
Prejuízo Líquido do Exercício Pela Correção Monetária Integral & $\mathbf{( 2 5 . 1 6 9 , 1 5 )}$ \\
\hline
\end{tabular}




\begin{tabular}{|r|r|r|r|r|r|r|r|r|r|}
\hline \multirow{2}{*}{ Data } & \multicolumn{3}{|c|}{ Entradas } & \multicolumn{3}{|c|}{ Saídas } & \multicolumn{2}{|c|}{ Saldo } \\
\hline & Quant. & Unit. R\$ Total R\$ & Quant. & Unit. R\$ & Total R\$ & Quant. & Unit. R\$ Total R\$ \\
\hline 31.01 .97 & 20 & 500,00 & $10.000,00$ & & & & 20 & 500,00 & $10.000,00$ \\
\hline 10.12 .97 & 18 & $1.111,00$ & $20.000,00$ & & & & 38 & 789,47 & $30.000,00$ \\
\hline 10.12 .97 & & & & 15 & 789,47 & $11.842,10$ & 23 & 789,47 & $18.157,90$ \\
\hline
\end{tabular}

\section{Movimentação do estoque - custo médio pela correção monetária integral}

\begin{tabular}{|c|c|c|c|c|c|c|c|c|c|}
\hline \multirow[b]{2}{*}{ Data } & \multicolumn{3}{|c|}{ Entradas } & \multicolumn{3}{|c|}{ Saídas } & \multicolumn{3}{|c|}{ Saldo } \\
\hline & Quant. & $\begin{array}{l}\text { Unit. } \\
\text { UMC'S }\end{array}$ & $\begin{array}{l}\text { Total } \\
\text { UMC'S }\end{array}$ & Quant. & $\begin{array}{l}\text { Unit. } \\
\text { UMC'S }\end{array}$ & $\begin{array}{l}\text { Total } \\
\text { UMC'S }\end{array}$ & Quant. & $\begin{array}{l}\text { Unit. } \\
\text { UMC'S }\end{array}$ & $\begin{array}{l}\text { Total } \\
\text { UMC'S }\end{array}$ \\
\hline 31.01 .97 & 20 & 591,50 & $11.830,12$ & & & & 20 & 591,50 & $11.830,12$ \\
\hline 10.12 .97 & 18 & 916,77 & $16.501,92$ & & & & 38 & 748,58 & $28.332,04$ \\
\hline \multirow[t]{2}{*}{10.12 .97} & & & & 15 & 745,58 & $11.183,70$ & 23 & 748,58 & $17.148,34$ \\
\hline & & & & $C P V$ & & $11.183,70$ & & & $17.148,34$ \\
\hline
\end{tabular}

\section{Empresa integralizadora S/A}

Demonstrativo da Correção Monetária de balanço para fins da integral findo em 31.12.97

(Valores em Reais)

\begin{tabular}{|c|c|c|c|c|c|c|}
\hline Discriminação & $\begin{array}{l}\text { Saldo } \\
\text { Inicial } \\
\mathbf{R} \$\end{array}$ & $\begin{array}{l}\text { Valor da } \\
\text { UMC para } \\
\text { conversão }\end{array}$ & $\begin{array}{l}\text { Quant. } \\
\text { UMC's }\end{array}$ & $\begin{array}{l}\text { Valor da } \\
\text { UMC final } \\
\text { p/correção }\end{array}$ & $\begin{array}{l}\text { Saldo Final } \\
\text { Corrigido }\end{array}$ & $\begin{array}{l}\text { Valor da } \\
\text { Correção } \\
\text { Monetária }\end{array}$ \\
\hline $\begin{array}{l}\text { Máquinas e Equipamentos } \\
\text { Ações de outras Empresas } \\
\text { (-) Depreciação Acumulada (*) } \\
\left(^{*}\right) \text { considerada proprocional }\end{array}$ & $\begin{array}{r}15.000,00 \\
10.000,00 \\
(1.250,00)\end{array}$ & $\begin{array}{l}0,8877 \\
0,9509 \\
0,8877\end{array}$ & $\begin{array}{r}16.897,601 \\
10.516,353 \\
(1.408,133)\end{array}$ & $\begin{array}{l}1,1500 \\
1,1500 \\
1,1500\end{array}$ & $\begin{array}{r}19.432,24 \\
12.093,81 \\
(1.619,35)\end{array}$ & $\begin{array}{l}4.432,24 \\
2.093,81 \\
(369,35)\end{array}$ \\
\hline Total Permanente & $23.750,00$ & & $26.005,821$ & & $29.906,70$ & $6.156,70$ \\
\hline $\begin{array}{l}\text { Capital Integralizado } \\
\text { Integralização do Capital - Adição }\end{array}$ & $\begin{array}{l}80.000,00 \\
20.000,00\end{array}$ & $\begin{array}{l}0,8287 \\
0,9509\end{array}$ & $\begin{array}{l}96.536,744 \\
21.032,706\end{array}$ & $\begin{array}{l}1,1500 \\
1,1500\end{array}$ & $\begin{array}{r}111.017,26 \\
24.187,61\end{array}$ & $\begin{array}{r}31.017,26 \\
4.187,61\end{array}$ \\
\hline Total Patrimônio Líquido & $100.000,00$ & & $117.569,450$ & & $135.204,87$ & $35.204,87$ \\
\hline Saldo (devedor) da CMB & & & & & & $(29.048,17)$ \\
\hline
\end{tabular}


AVP - FORNECEDOR

$$
\operatorname{AVP}=\frac{1}{(1+i) n}
$$

NA DATA DA TRANSAÇÃO: $\mathbf{1 0 . 1 2 . 9 7}$

$\mathrm{R} \$ 20.000,00 / 1,10=\mathrm{R} \$ 18.181,81 / 1,1018=$ UMCS $16.501,9150$

NA DATA DO ENCERRAMENTO DO BALANÇO: 31.12 .97

$R \$ 20.000,00 / 1,0322=R \$ 19.376,09$

CÁLCULO DA DESPESA FINANCEIRA

$\mathrm{R} \$ 19.376,09-\mathrm{R} \$ 18.181,81=\mathrm{R} \$ 1.194,28 /$ UMC $1,1500=$ UMC's $1.038,5000$

CÁLCULO DO GANHO DO FORNECEDOR

$R \$ 19.376,09 / 1,1018(-) R \$ 19.376,09 / 1,1500=737,0800$ UMC's

$17.585,85$ UMC's (-) $16.848,77$ UMC's $=737,0800$ UMC's

2 - Cálculo do ajuste a valor presente - AVP

$$
\text { AVP - CLIENTE } \quad \text { AVP }=\frac{1}{(1+i) n}
$$

NA DATA DA TRANSAÇÃO: $\mathbf{1 0 . 1 2 . 9 7}$

$\mathrm{R} \$ 50.000,00 / 1,12=\mathrm{R} \$ 44.642,85 / 1,1018=$ UMC's $40.518,1067$

NA DATA DO ENCERRAMENTO DO BALANÇO: $\mathbf{3 1 . 1 2 . 9 7}$

$R \$ 50.000,00 / 1,03458=R \$ 48.328,79$

CÁLCULO DA RECEITA FINANCEIRA

$R \$ 48.328,79-R \$ 44.642,85=R \$ 3.685,94 / 1,1500=3.205,1652$ UMC's

\section{CÁLCULO DA PERDA COM O CLIENTE}

$\mathrm{R} \$ 48.328,79 / 1,1018$ (-) R\$ 48.328,79/1,1500=1.838,4523 UMC's

$43.863,4870$ UMC's (-) 42.025,0347 UMC's $=1.838,4523$ UMC's.

Demonstração Gráfica do AVP - Outro exemplo

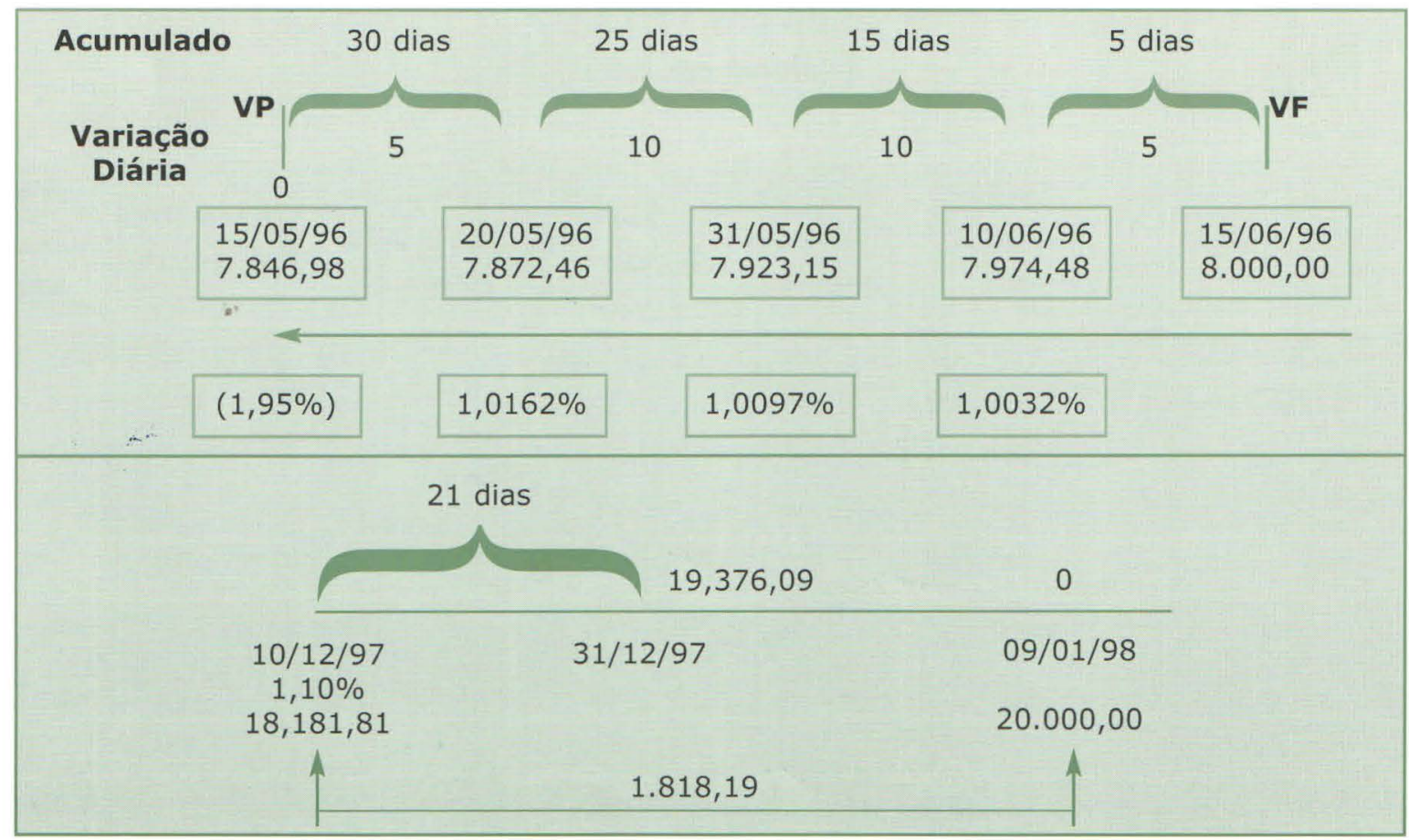




\section{CÁlCULO DO A.V.P. DADO NO EXERCÍCIO (PELA HP 12C)}

AVP - FORNECEDOR - 01

1,10 ENTER

10 ENTER

30 DIVIDE

yx

1-

$100 \mathrm{x}$

$=3,228012$
AVP - CLIENTE - 02

1,12 ENTER

9 ENTER

30 DIVIDE

yx

$1-$

$100 \mathrm{x}$

$=3,458316$

\section{OUTROS EXEMPLOS:}

Valor Futuro $=\mathrm{FV}=\mathrm{R} \$ 717,69$

Valor Presente $=P V=R \$ 542,68$

$\mathrm{R} \$ 717,69 \mathrm{FV}$

$32,25 \mathrm{i}$

$1 \mathrm{n}$

apertar PV

\section{CÁLCULO DOS JUROS COMPOSTOS (PELA HP 12C)}

\section{EXEMPLO:}

$\mathrm{i}=15 \%$ ao mês

$\mathrm{N}=2$ meses

Valor absoluto $=100$

100 PV

$15 \mathrm{i}$

$2 n$

APERTAR FV E TIRAR 100

Empresa integralizadora S/A

Demonstração das origens e aplicações de recursos

do exercício encerrado em 31.12.97

(Valores em Reais)

$\begin{array}{rr}31.12 .97 & 31.12 .97 \\ \text { LEG. SOC. } & \text { C.M.I. }\end{array}$

\section{ORIGENS DE RECURSOS}

Prejuízo líquido do Exercício

(+) Depreciação

Financiamento do Capital de Giro

Integralização de Capital em Moeda

TOTAL DÁS ORIGENS
$(4.917,10)$

$1.250,00$

$40.000,00$

$20.000,00$

$56.332,90$
$(25.169,15)$

$1.619,35$

$40.000,00$

$24.187,61$

\section{APLICAÇÕES DE RECURSOS}

Depósito Judicial - PIS s/ Faturamento

Aquisição de Investimento - Ações de outras Cias

Aquisição de Imobilizado

TOTAL DAS APLICAÇÕES

Aumento (Diminuição) Capital Circ. Líquido
$18.000,00$

$10.000,00$

$15.000,00$

$43.000,00$

$13.332,90$
$18.000,00$

$12.093,81$

$19.432,24$

49.526,05

$(8.888,24)$ 


\section{Demonstração da variação do capital circulante líquido}

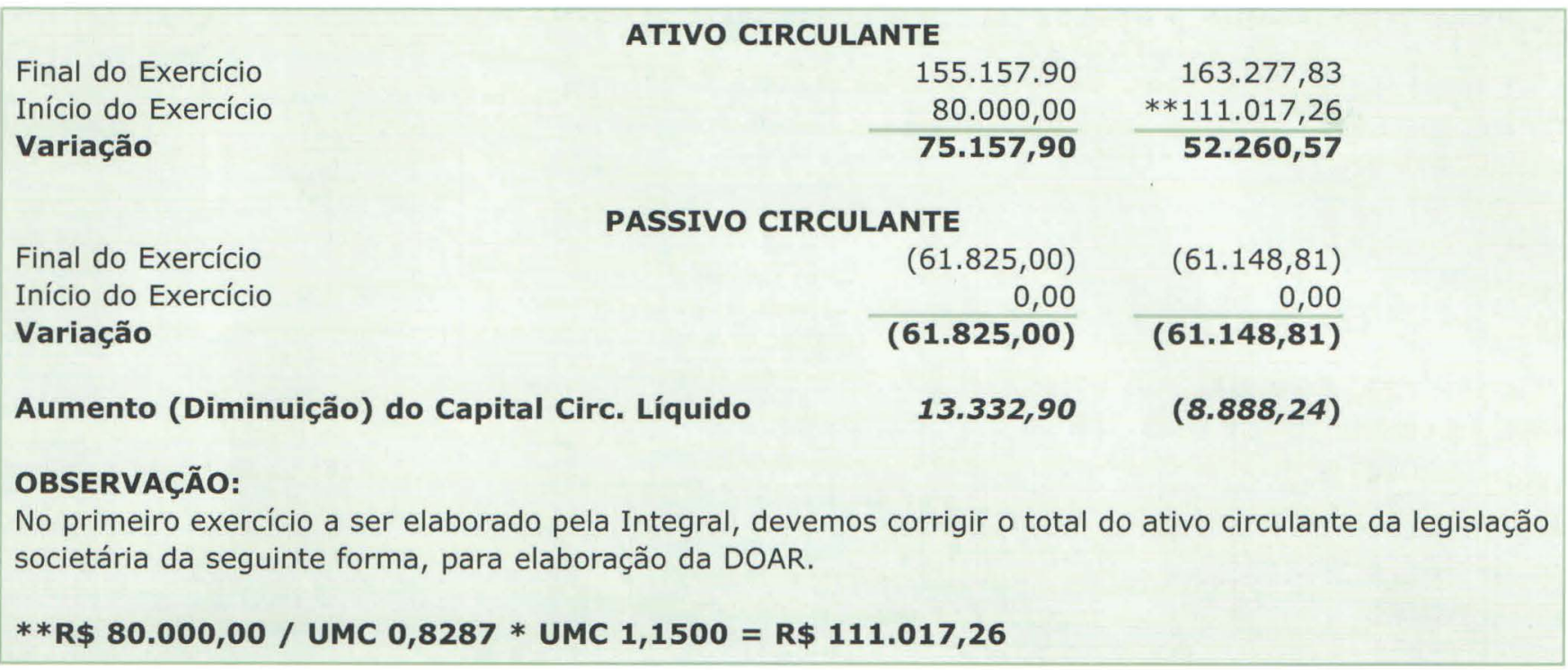

\section{Conclusão}

Podemos observar que a variação da UMC utilizada no início do exercício, em 31/12/1996, e no final de exercício, em 31/12/1997, variou 38,77\%. Prejuízo pela legislação societária no valor de $\mathrm{R} \$$ 4.917,10, e pela integral de $\mathrm{R} \$ 25.169,15$, demonstrou uma variação de $411,86 \%$.

A estrutura de cada empresa, ou seja, seus ativos e passivos monetários expostos à inflação, aliados à política de juros extorsivos praticados nas transações econômicas de nosso país, podem gerar grandes impactos no resultado contábil e financeiro das empresas. Por esse motivo, especialistas e grandes mestres de nossa área, de renomadas escolas, constantemente publicam artigos e simulações a respeito destas distorções provocadas pelas incontinências da economia política de nosso país.

Cabe ao contabilista ficar atento e zelar pela informação contábil da entidade onde atua, pois somos os mentores da maximização da riqueza, denominada de lucro.

\section{REFERÊNCIAS BIBLIOGRÁFICAS}

ALMEIDA, Marcelo Cavalcanti. Correção Monetária Integral das Demonstrações Financeiras: de acordo com a Instrução CVM $n^{\underline{0}}$ 64, de 19/05/87. São Paulo: Atlas, 1987.

COELHO, Juarez Ferreira. O Uso das Técnicas da Correção Monetária Integral na Conversão das Demonstrações em Moeda Estrangeira. São Paulo, 1996. Dissertação (Mestrado) - Pontifícia Universidade Católica - SP.

COOPERS \& LYBRAND. Relatórios Financeiros e Inflação: Gerenciamento Eficaz com Moeda Forte. São Paulo: Coopers \& Lybrand, 1990.

CUNHA, Antonio Quinta. Contabilização da Inflação na Empresa Brasileira - da Lei 6404/76 à Instrução 64/87 da CVM. São Paulo, 1988. Dissertação (Mestrado) - Fundação Getúlio Vargas.

FALCÃO, Eduardo de Aquino. Divulgação em Demonstrações Financeiras de Companhias Abertas. São Paulo, 1993. Dissertação (Mestrado) - Faculdade de Economia e Administração/USP.

FIPECAFI. Aprendendo Contabilidade em Moeda Constante. São Paulo: Atlas, 1994.

SANTOS, Ariovaldo dos. Alguns Efeitos da Utilização de Índices Inadequados na Correção dos Balanços de Empresas Estrangeiras no Brasil. São Paulo, 1993. Tese (Doutorado) - Faculdade de Economia e Administração/USP. 